\title{
Ultrafast Spin Density Wave Transition in Chromium Governed by Thermalized Electron Gas
}

\author{
C. W. Nicholson, ${ }^{1, *}$ C. Monney, ${ }^{2}$ R. Carley, ${ }^{3, \dagger}$ B. Frietsch,${ }^{3,4}$ J. Bowlan, ${ }^{3, *}$ M. Weinelt, ${ }^{4}$ and M. Wolf ${ }^{1}$ \\ ${ }^{1}$ Department of Physical Chemistry, Fritz-Haber-Institut of the Max Planck Society, Faradayweg 4-6, Berlin 14915, Germany \\ ${ }^{2}$ Department of Physics, University of Zurich, Winterthurerstrasse 190, 8057 Zurich, Switzerland \\ ${ }^{3}$ Max-Born-Institut, Max-Born-Strasse 2A, 12489 Berlin, Germany \\ ${ }^{4}$ Fachbereich Physik, Freie Universitaet Berlin, Arnimallee 14, 14195 Berlin, Germany
}

(Received 14 April 2016; published 20 September 2016)

\begin{abstract}
The energy and momentum selectivity of time- and angle-resolved photoemission spectroscopy is exploited to address the ultrafast dynamics of the antiferromagnetic spin density wave (SDW) transition photoexcited in epitaxial thin films of chromium. We are able to quantitatively extract the evolution of the SDW order parameter $\Delta$ through the ultrafast phase transition and show that $\Delta$ is governed by the transient temperature of the thermalized electron gas, in a mean field description. The complete destruction of SDW order on a sub-100 fs time scale is observed, much faster than for conventional charge density wave materials. Our results reveal that equilibrium concepts for phase transitions such as the order parameter may be utilized even in the strongly nonadiabatic regime of ultrafast photoexcitation.
\end{abstract}

DOI: 10.1103/PhysRevLett.117.136801

In ultrafast dynamics, the transfer of energy from a short laser pulse can lead to the population of excited states [1-3], changes in magnetic ordering [4,5], and even electronic or structural phase transitions [6-9]. In many cases a transient increase of the electronic temperature occurs that may be tracked, for example, by time- and angle-resolved photoemission spectroscopy (trARPES) $[1,10,11]$. An open question is still to what extent the electronic temperature alone can be said to govern ultrafast changes, particularly for phase transitions, due to the strongly nonadiabatic nature of pump-probe experiments and the possibility of exciting nonthermal electron distributions on short time scales. Such a description is further complicated in many correlated materials such as high- $T_{c}$ superconductors, charge density waves (CDWs), and ferromagnets, in which lattice degrees of freedom play an important role. In the case of CDWs, the periodic motion of the atomic cores (phonons) can lead to a periodic opening and closing of the spectral gap [6,12-14] independent of the temperature of the electronic system. In ultrafast demagnetization of ferromagnets, a bottle neck for the transition is the transfer of angular momentum, which proceeds through the lattice [15] meaning that a hot electron system may be necessary, but is not sufficient to drive the system from one magnetic phase to another. In contrast, the ordering in spin density waves stems directly from electronic correlations [16] and thus offers an opportunity to study the dynamics of a phase transition in which the role of the lattice is minimized. This allows a more stringent test of the role played by the electronic temperature in driving materials from one phase to another under nonequilibrium conditions.
$\mathrm{Cr}$ famously undergoes a transition to an antiferromagnetic-SDW phase [17]. Although the SDW in $\mathrm{Cr}$ has been widely studied [17-21], very few studies of the timedomain dynamics exist [22-24], none of which directly addresses the electronic structure.

In this Letter we report the first time-resolved ARPES investigation of the SDW transition in Cr. We directly observe the ultrafast disappearance and recovery of the SDW-derived electronic structure following pulsed infrared excitation. The ultrafast dynamics of the electronic structure are simulated with a mean field model in order to disentangle intrinsic SDW dynamics from other nonequilibrium effects. We find that the order parameter of the SDW is governed by the transient electronic temperature, implying an intimate link between electronic temperature and spin ordering as the driving mechanism of the SDW in Cr. Our results demonstrate that equilibrium thermodynamic concepts can still survive on ultrashort time scales, in cases where the temperature of a single subsystem governs the behavior of an ordered phase.

$\mathrm{Cr}(110)$ films were grown epitaxially on a clean W(110) crystal at room temperature at a pressure of $1 \times 10^{-10} \mathrm{mbar}$, and then annealed to $600^{\circ} \mathrm{C}$. A film thickness of $7 \mathrm{~nm}$ was produced by reference to the Low Energy Electron Diffraction (LEED) and ARPES phase diagram [20]. For the pump-probe measurements, shown schematically in Fig. 1(a), a linear polarized $1.5 \mathrm{eV}$ pump and $40 \mathrm{eV}$ extreme ultraviolet (XUV) probe were utilized to collect snapshots of the electronic structure. A detailed description of our high-harmonic generation-based trARPES setup is presented elsewhere [25]. Because of the optical-absorption depth of $30 \mathrm{~nm}$ at the pump wavelength [26], the entire thin film is excited nearly homogeneously. The combined time 
(a)

(b)
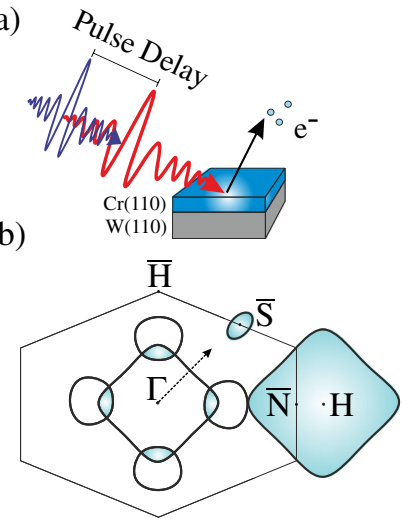

(c)

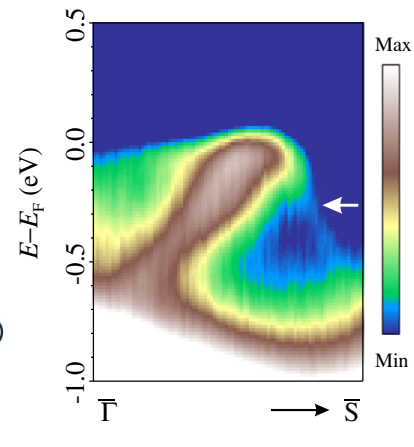

FIG. 1. (a) Schematic pump-probe experiment of the $\operatorname{Cr}(110)$ thin film. (b) Schematic Fermi surface and surface Brillouin zone of Cr. Electron pockets are contained within solid black lines while hole pockets are colored cyan. The hexagon marks the (110) surface projected Brillouin zone. (c) Electronic structure of $\mathrm{Cr}(110)$ thin film measured along the dotted arrow in Fig. 1(b). The spin density wave (SDW) band is marked by a white arrow.

resolution of the experiment was measured to be $130 \mathrm{fs}$. Unless otherwise stated, a fluence of $0.8 \mathrm{~mJ} \mathrm{~cm}^{-2}$ and an $s$-polarized pump were selected to maximize the pumpprobe signal while avoiding significant space charge. ARPES measurements were carried out with a hemispherical electron analyzer (SPECS) at $100 \mathrm{~K}$, well below the surface SDW transition temperature of $440 \mathrm{~K}$ [18]. All results presented here are from the band at the Fermi level $E_{F}$ along the $\bar{\Gamma}-\bar{S}$ direction marked in Fig. 1(b).

Fig. 1c shows the electronic structure of $\mathrm{Cr}$ in the SDW phase measured with He I radiation. The data are plotted in a false colour log scale to highlight weak features. An electron band is seen to disperse towards the Fermi level $\left(E_{F}\right)$ from $\bar{\Gamma}$ towards the $\bar{S}$ point. As the band approaches $E_{F}$ it bends away and continues again to higher binding energies. This feature with weak spectral weight is a direct result of the SDW periodicity that results in a band renormalization via back folding [20]. The fact that we observe the renormalized dispersion in our data confirms the high quality of our $\mathrm{Cr}$ film preparation. A holelike band with maxima at $-0.7 \mathrm{eV}$ binding energy is also derived from $\mathrm{Cr}$ states [not shown in Fig. 1(c)].

The same spectrum obtained with pulsed XUV radiation is presented in Fig. 2(a). As expected in the SDW phase the renormalized band is observed, though it is weaker than in the He-lamp measurements due to the lower energy resolution of the high harmonics source of $200 \mathrm{meV}$. Upon excitation with the pump laser pulse, the dispersion is observed to change. As shown for $200 \mathrm{fs}$ after optical excitation, the dispersion linearly crosses the Fermi level, as in the paramagnetic phase $[18,20]$, while spectral weight is removed from the renormalized band. These changes are highlighted by the difference image in Fig. 2(c) in which one clearly sees a strong reduction (blue) of spectral weight in the SDW band region, which is gained above the Fermi (a)
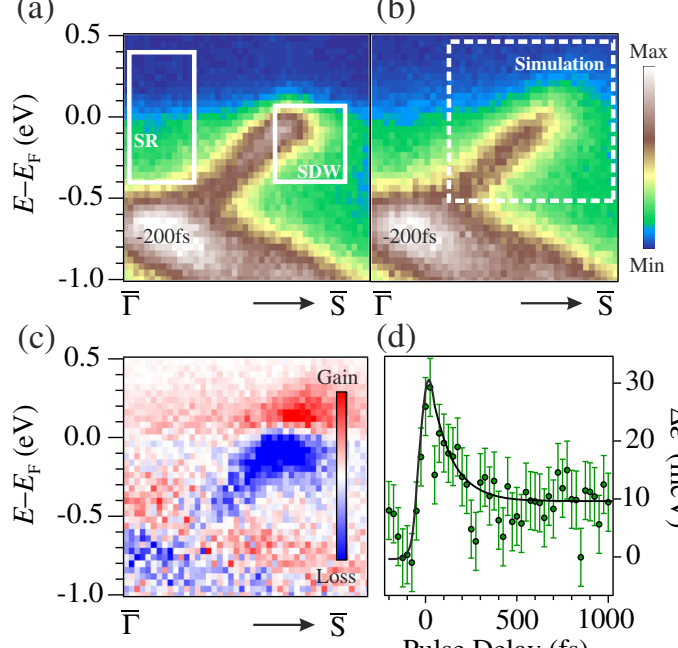

(d)

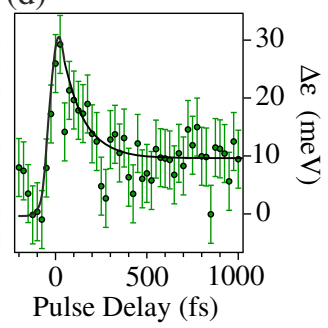

FIG. 2. ARPES images obtained with $40 \mathrm{eV}$ probe energy (a) in thermal equilibrium at a delay of $-200 \mathrm{fs}$ (XUV probe pulse before pump pulse) and (b) following optical excitation at $200 \mathrm{fs}$ delay. The dotted box highlights the area considered in the simulation as presented in Fig. 4(a). (c) Difference image between the images shown in (a) and (b). In the false color plot red and blue marks intensity increase and decrease, respectively. (d) Transient rigid shift of the energy distribution curves (EDCs). The solid black line is a guide to the eye.

level (red). Additionally, between the SDW band and $\bar{\Gamma}$, intensity is gained above $E_{F}$ due to the broadening of the Fermi-Dirac distribution of the metallic states from a surface resonance [21].

Also visible in Fig. 2(c) at $200 \mathrm{fs}$ is a rigid shift of the entire electronic structure towards higher kinetic energies. A shift of almost $30 \mathrm{meV}$ is observed, which is the same, within experimental errors, as the observed shift of the Fermi level. The dynamics of this shift over the time delay of the experiment are given in Fig. 2(d), revealing a rapid peak followed by recovery to a new equilibrium value by around 500 fs. Such dynamics are not typical of probeinduced space charge effects. The origin of this shift may be due to out-of-equilibrium chemical potential shifts, or a change of the potential barrier at the sample surface induced by the transient electronic temperature. For simplicity we treat it purely phenomenologically in the following analysis.

EDCs from the metallic surface resonance region [box SR in Fig. 2(a)] are presented in Fig. 3(a) at different pulse delays. By fitting a Fermi-Dirac distribution convolved with the instrumental resolution, the transient electronic temperature $\left(T_{e}\right)$ may be extracted. We note that within our time resolution of $130 \mathrm{fs}$ we always observe a thermal distribution of electrons. Figure 3(b) displays the evolution of $T_{e}$ up to $1000 \mathrm{fs}$ following excitation. After a rapid increase to $1200 \mathrm{~K}$ close to $0 \mathrm{fs}, T_{e}$ decreases to a new quasiequilibrium value after 800 fs. 

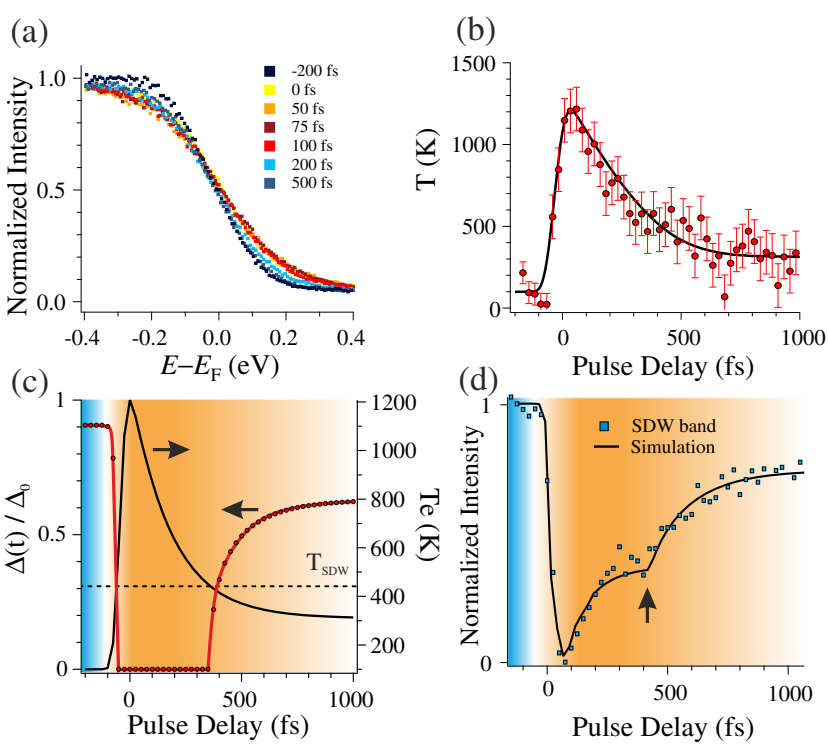

FIG. 3. (a) Fermi distribution extracted from region SR indicated in Fig. 2(a) at various pulse delays. (b) Electronic temperature extracted from the Fermi distribution as a function of pulse delay. The solid black line is a guide to the eye. (c) Electronic temperature (solid black line) and associated order parameter (red markers) calculated as a function of pulse delay. The dotted line marks the SDW transition temperature. (d) Dynamics of the normalized SDW band intensity in the region marked SDW in Fig. 2(a) for both experiment (markers) and simulation (solid line). An arrow marks the point at which $\Delta$ transitions from 0 to a finite value. The color background in (c) and (d) highlights the region where the SDW is in the ground (blue) and excited (orange) state.

In order to investigate the quantitative effect of $T_{e}$ on the SDW electronic structure, we utilize results from a mean field model, which may be applied to BCS superconductors and density waves $[27,28]$, in order to describe the SDW as a renormalized dispersion with a gap. In such a model, the poles of the Green's function give the quasiparticle dispersion, which may be written as $E_{ \pm}=$ $\pm \sqrt{\epsilon(k)^{2}+\Delta^{2}}$, where $\epsilon$ is the bare band dispersion. This renormalized dispersion has two branches separated by a gap determined by $\Delta$. The temperature dependence of the SDW order parameter $(\Delta)$ is given by

$$
\Delta\left(T_{e}\right)=\Delta_{0}\left(1-\frac{T_{e}}{T_{\mathrm{SDW}}}\right)^{\beta},
$$

where $\Delta_{0}$ is half the gap measured by photoemission, and $T_{\mathrm{SDW}}=440 \mathrm{~K}$ is the surface phase transition temperature of the SDW. This relation $(\beta=0.38)$ has been determined in the ARPES study of [18]. The spectral weight in the renormalized bands is given by the coherence factors $u_{ \pm}^{2}=\left(1+\epsilon / E_{ \pm}\right) / 2$. Further details may be found in the Supplemental Material [29]. In the experiment each time delay is associated with a particular $T_{e}$; hence, we introduce time resolution into the simulation by producing snapshots of the electronic structure at the experimentally determined $T_{e}$. The rigid shift dynamics are added phenomenologically.

The response of the order parameter to the transient $T_{e}$ is shown in Fig. 3(c), and compared with $T_{e}$. In the region where $T_{e}>T_{C}, \Delta$ goes to 0 , reflecting the closing of the SDW gap. Once $T_{e}$ drops below $T_{C}, \Delta$ again becomes finite and assumes a value given by $T_{e}$. The resulting simulated spectral response of the renormalized SDW band is shown in Fig. 3(d) as a solid black line. The energy and momentum region considered in the simulation is marked in Fig. 4 (left panel) by a white box, which is equivalent to that used to extract the response of the data, as marked in Fig. 2(a). Following excitation, two distinct regions of recovery are evident $(t>100 \mathrm{fs})$. The first rapid increase in intensity is due to the relaxation of the rigid band shift presented in Fig. 2(d), which shifts the electronic structure relative to the fixed region of interest (ROI) [30]. In our simulation it is found that the rigid shift dynamics and the dynamics of the Fermi distribution (modeled by the SDW density of states multiplied by the Fermi function) give the same qualitative response. Underlying the rigid shift dynamics are the dynamics of the order parameter, which is saturated to 0 directly following excitation due to the large $T_{e}$. As shown in Fig. 3(c), the order parameter plateaus in the time region $-50 \mathrm{fs}<t<400 \mathrm{fs}$ but becomes finite at longer time delays. Once $\Delta>0$, spectral

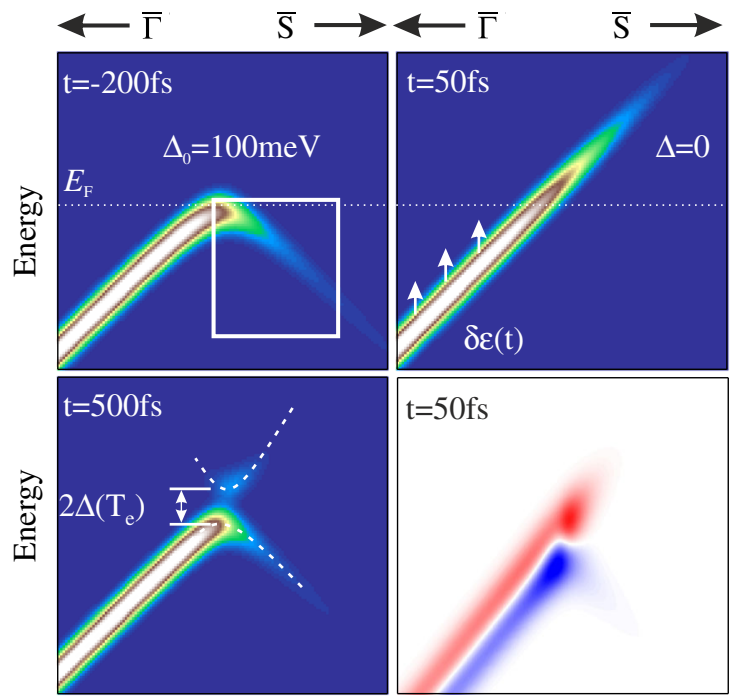

FIG. 4. Snapshots of the simulated electronic structure at -200 , 50, and $500 \mathrm{fs}$ in the region highlighted in Fig. 2(b). Following pump excitation both the order parameter and the rigid shift $(\delta \epsilon)$ change transiently, leading to a reduction of spectral weight in the analyzed region. Initial excitation leads to the transition to the paramagnetic phase (50 fs), which relaxes into the SDW phase once $T_{e}<T_{\mathrm{SDW}}(500 \mathrm{fs})$. Dotted lines in the bottom left panel are guides to the eye following the dispersion in both branches of the renormalized dispersion. The difference image between -200 and $50 \mathrm{fs}$ highlights the redistrubution of intensity as observed in the experiment [Fig. 2(c)]. 
weight is transferred into the renormalized SDW band, resulting in a shoulder in the recovery dynamics, marked by the arrow in Fig. 3(d). The simulated response is compared to the experimental data from the region of the SDW band [box SDW in Fig. 2(a)] and shows good agreement.

Snapshots from the simulation presented in Fig. 4 summarize the effects taking place in the electronic structure. For a given pulse delay, the magnitude of the order parameter is determined by $T_{e}$, which therefore defines both the size of the gap and the spectral weight of the renormalized band. In addition the rigid band shift results in a reduction of intensity for a fixed region of the electronic structure. The separation of the two effects (the rigid band shift and order parameter dynamics) is presented explicitly in Fig. 2 of the Supplemental Material.

Further evidence that $\Delta$ is solely governed by $T_{e}$ comes from low fluence data [29]. At a fluence of $0.2 \mathrm{~mJ} \mathrm{~cm}^{-2}$ the recovery dynamics exhibit only a single relaxation time scale as a result of a mixture of the rigid shift dynamics and the always finite, but varying, order parameter. This is consistent with the extracted $T_{e}$ curve, which reveals the electronic system does not reach the SDW transition temperature, i.e., $T_{e}<T_{\mathrm{SDW}}$ for all times. Thus, $\Delta$ has a finite value for all time delays at this fluence, and the system is not driven into the paramagnetic phase. This corroborates the idea that upon ultrafast optical excitation the melting and recovery of the SDW is driven by a purely electronic mechanism and that we can indeed quantitatively track $\Delta$ through the ultrafast phase transition.

The disappearance of the SDW signature-the renormalized band-implies the electron gas no longer experiences the SDW spin-ordering potential, which results in the disappearance of the long-range spin ordering. Since AFM ordering is present only in the SDW phase, once the SDW is removed, the magnetic order also disappears on a sub100 fs time scale, as the sample transitions to the paramagnetic phase. We thus conclude that the SDW is directly driven by the transient $T_{e}$ and that even on ultrashort time scales the transient heating of the electrons drives the spin ordering of the SDW transition. The lattice plays a limited role in the SDW transition, acting as a heat sink allowing the heated electron gas to cool and the SDW ordering to reemerge.

In summary, we have used trARPES at XUV energies to investigate the excitation and recovery of the SDW in $\mathrm{Cr}(110)$ thin films on ultrafast time scales. We find that the transition to the paramagnetic state occurs promptly within the pump-pulse duration. In addition, we show that the order parameter $\Delta$ follows the electronic temperature $T_{e}$, which governs both the closing and reopening of the SDW gap. This therefore suggests that the electrons form a quasiequilibrium with the spin order. This shows that concepts from thermodynamic equilibrium can survive in the ultrafast regime for phases governed by the temperature of a single subsystem, even if this is not equilibrated with other degrees of freedom, e.g., phonons. Such a concept should not only be true for electrons. Therefore, we speculate that such a mean field approach may be applicable to other ordered phases that are governed by a single subsystem temperature.

C. M. acknowledges support by the Swiss National Science Foundation under Grant NO. PZ00P2_154867, as well as by the Alexander von Humboldt Foundation.

*nicholson@fhi-berlin.mpg.de

†Present address: European XFEL GmbH, Notkestrasse 85, 22607 Hamburg, Germany.

"Present address: CINT, Los Alamos National Laboratory, Albuquerque, New Mexico 87185-1315, USA.

[1] I. Gierz, J. C. Petersen, M. Mitrano, C. Cacho, I. C. E. Turcu, E. Springate, A. Stöhr, A. Köhler, U. Starke, and A. Cavalleri, Nat. Mater. 12, 1119 (2013).

[2] A. Grubišić Čabo, J. a. Miwa, S. S. Grønborg, J. M. Riley, J. C. Johannsen, C. Cacho, O. Alexander, R. T. Chapman, E. Springate, M. Grioni, J. V. Lauritsen, P. D. C. King, P. Hofmann, and S. Ulstrup, Nano Lett. 15, 5883 (2015).

[3] J. A. Sobota, S. Yang, J. G. Analytis, Y. L. Chen, I. R. Fisher, P. S. Kirchmann, and Z.-X. Shen, Phys. Rev. Lett. 108, 117403 (2012).

[4] I. Radu, K. Vahaplar, C. Stamm, T. Kachel, N. Pontius, H. A. Dürr, T. A. Ostler, J. Barker, R. F. L. Evans, R. W. Chantrell, A. Tsukamoto, A. Itoh, A. Kirilyuk, T. Rasing, and A. V. Kimel, Nature (London) 472, 205 (2011).

[5] B. Frietsch, J. Bowlan, R. Carley, M. Teichmann, S. Wienholdt, D. Hinzke, U. Nowak, K. Carva, P. M. Oppeneer, and M. Weinelt, Nat. Commun. 6, 8262 (2015).

[6] F. Schmitt, P. S. Kirchmann, U. Bovensiepen, R. G. Moore, L. Rettig, M. Krenz, J.-H. Chu, N. Ru, L. Perfetti, D. H. Lu, M. Wolf, I. R. Fisher, and Z.-X. Shen, Science 321, 1649 (2008).

[7] T. Rohwer, S. Hellmann, M. Wiesenmayer, C. Sohrt, A. Stange, B. Slomski, A. Carr, Y. Liu, L. M. Avila, M. Kalläne, S. Mathias, L. Kipp, K. Rossnagel, and M. Bauer, Nature (London) 471, 490 (2011).

[8] P. Beaud et al., Nat. Mater. 13, 923 (2014).

[9] L. Waldecker, T. A. Miller, M. Rudé, R. Bertoni, J. Osmond, V. Pruneri, R. E. Simpson, R. Ernstorfer, and S. Wall, Nat. Mater. 14, 991 (2015).

[10] Y. H. Wang, D. Hsieh, E. J. Sie, H. Steinberg, D. R. Gardner, Y. S. Lee, P. Jarillo-Herrero, and N. Gedik, Phys. Rev. Lett. 109, 127401 (2012).

[11] A. Crepaldi, B. Ressel, F. Cilento, M. Zacchigna, C. Grazioli, H. Berger, P. Bugnon, K. Kern, M. Grioni, and F. Parmigiani, Phys. Rev. B 86, 205133 (2012).

[12] L. Rettig, R. Cortés, J.-H. Chu, I. R. Fisher, F. Schmitt, R. G. Moore, Z.-X. Shen, P. S. Kirchmann, M. Wolf, and U. Bovensiepen, Nat. Commun. 7, 10459 (2016).

[13] L. X. Yang, G. Rohde, T. Rohwer, A. Stange, K. Hanff, C. Sohrt, L. Rettig, R. Cortés, F. Chen, D. L. Feng, T. Wolf, B. Kamble, I. Eremin, T. Popmintchev, M. M. Murnane, H. C. Kapteyn, L. Kipp, J. Fink, M. Bauer, U. Bovensiepen, and K. Rossnagel, Phys. Rev. Lett. 112, 207001 (2014). 
[14] E. Papalazarou, J. Faure, J. Mauchain, M. Marsi, A. TalebIbrahimi, I. Reshetnyak, A. Van Roekeghem, I. Timrov, N. Vast, B. Arnaud, and L. Perfetti, Phys. Rev. Lett. 108, 256808 (2012).

[15] J. Stöhr and H. C. Siegmann, Magnetism: From Fundamentals to Nanoscale Dynamics (Springer-Verlag, Berlin, 2006).

[16] A. W. Overhauser, Phys. Rev. 128, 1437 (1962).

[17] E. Fawcett, Rev. Mod. Phys. 60, 209 (1988).

[18] J. Schäfer, E. Rotenberg, G. Meigs, S. D. Kevan, P. Blaha, and S. Hüfner, Phys. Rev. Lett. 83, 2069 (1999).

[19] E. Fawcett, H. L. Alberts, V. Y. Galkin, D. R. Noakes, and J. V. Yakhmi, Rev. Mod. Phys. 66, 25 (1994).

[20] E. Rotenberg, B. K. Freelon, H. Koh, A. Bostwick, K. Rossnagel, A. Schmid, and S. D. Kevan, New J. Phys. 7, 114 (2005).

[21] E. Rotenberg, O. Krupin, and S. D. Kevan, New J. Phys. 10, 023003 (2008).

[22] H. Hirori, T. Tachizaki, O. Matsuda, and O. B. Wright, Phys. Rev. B 68, 113102 (2003).

[23] A. Singer, M. J. Marsh, S. H. Dietze, V. Uhlír, Y. Li, D. A. Walko, E. M. Dufresne, G. Srajer, M. P. Cosgriff, P. G. Evans, E. E. Fullerton, and O. G. Shpyrko, Phys. Rev. B 91, 115134 (2015).
[24] A. Singer, S. K. K. Patel, R. Kukreja, V. Uhlír, J. Wingert, S. Festersen, D. Zhu, J. M. Glownia, H. T. Lemke, S. Nelson, M. Kozina, K. Rossnagel, M. Bauer, B. M. Murphy, O. M. Magnussen, E. E. Fullerton, and O. G. Shpyrko, Phys. Rev. Lett. 117, 056401 (2016).

[25] B. Frietsch, R. Carley, K. Döbrich, C. Gahl, M. Teichmann, O. Schwarzkopf, P. Wernet, and M. Weinelt, Rev. Sci. Instrum. 84, 075106 (2013).

[26] A. D. Rakic, A. B. Djurisic, J. M. Elazar, and M. L. Majewski, Appl. Opt. 37, 5271 (1998).

[27] G. D. Mahan, Many-Particle Physics, 3rd ed. (Pernum, New York, 2000).

[28] G. Grüner, Density Waves in Solids (Perseus Publishing, New York, 1994).

[29] See Supplemental Material at http://link.aps.org/ supplemental/10.1103/PhysRevLett.117.136801 for further details.

[30] In principle, such an effect may be removed by considering a ROI large enough to encompass both the bands plus the shift. However, doing so means the ROI includes the region in which the Fermi distribution is transiently broadened and thus a transient change of intensity is also observed here due to changing thermal distribution. 